experience. Large studies or meta-analyses with effective control for confounders, adequate exposure assessment, and clear case definition are (ideally) required.

Results Although epidemiological studies may show consistent evidence of an increased health risk from an occupational exposure, definitions of exposure are often unsuitable for converting into an appropriate exposure schedule for a compensation scheme. Direct measurements of occupational exposure are usually scarce and not available for individual workers; both claimants, perhaps, assessors would find these measurements difficult to access and use. Exposures defined by industry or job title, particularly with a qualifying time-period, although perceived by epidemiologists as rather simplistic, are more straightforward to use, e.g. 'osteoarthritis in miners who have worked 10 or more years underground'. If quantitative exposure measurements are unavailable, epidemiologists often define qualitative metrics such as 'high', 'medium', or 'low' or develop more complex semi-qualitative exposure metrics such as 'exposed or not' at a given level of certainty or probability; intensity of exposure based on expert judgement of proximity to the substance and effectiveness of control procedures. Application of these in a compensation scheme may be problematical.

Discussion Epidemiology plays a vital role in ensuring workers are compensated for work-related ill-health. Epidemiological study design, exposure metrics and primary and subsidiary analyses should be tailored to directly support compensation schemes.

\section{O2C.2 DOES REGION OF RESIDENCE MATTER FOR RETURN-TO- WORK AFTER WORK-RELATED INJURY? A COMPARATIVE ANALYSIS OF SIX CANADIAN WORKERS' COMPENSATION JURISDICTIONS}

${ }^{1}$ Robert Macpherson*, ${ }^{1}$ Hui Shen, ${ }^{1}$ Mieke Koehoorn, ${ }^{2}$ Benjamin Amick, ${ }^{3}$ Alex Collie,
2,3 Peter Smith, ${ }^{1,2}$ Christopher McLeod. ${ }^{1}$ University of British Columbia, Vancouver, Canada;
${ }^{2}$ Institute for Work and Health, Toronto, Canada; ${ }^{3}$ Monash University, Melbourne, Australia

10.1136/OEM-2019-EPI.42

Objectives To investigate regional differences in return-to-work following work-related injury and whether these differences persist after adjusting for individual characteristics.

Methods Workers' compensation claims from six Canadian provinces were used to create comparable cohorts of workers aged 15-80 with a work-related injury resulting in at least one disability day from 2011 to 2015. Workers' residential postal codes were mapped to Census standard geographic units to categorize workers into six regions representing decreasing urban density and metropolitan influence (ranging from large urban areas of 100,000+peoople to rural areas of $<10000$ people with no metropolitan influence). Cox regression models were used to estimate the effect of urban-rural residence on the likelihood of injured workers transitioning off work disability benefits within one-year post-injury, adjusting for confounders, including provincial compensation jurisdiction. Models were stratified by industry sectors.

Results The cohort included 746029 work disability claims, of which the majority resided in large urban areas (69\%). Unadjusted models showed that workers residing in smaller urban and rural areas had a lower likelihood of transitioning off work disability benefits compared to those in large urban areas. Urban-rural differences persisted in adjusted models (e.g. $\mathrm{HR}=0.9195 \% \mathrm{CI} 0.89,0.94$ for workers in rural areas with no metropolitan influence). Industry-stratified models showed that greater differences existed between urban and rural places of residence for workers in the transportation and construction sectors, and smaller differences for workers in the health care and manufacturing sectors.

Conclusions The main finding suggests that injured workers in more rural areas face barriers in returning to work and that workers' compensation resources may need to be allocated to address these regional disparities. Future research will incorporate both individual and regional-level variables in a multilevel model framework to identify the characteristics that are the most important in explaining variability in work disability duration.

\section{O2C.3 WHAT PREDICTS A SECONDARY ABSENCE FOLLOWING RETURN TO WORK AMONG WORKERS' COMPENSATION CLAIMANTS IN VICTORIA? RESULTS FROM A LONGITUDINAL COHORT}

${ }^{1}$ Peter Smith*. 'Institute for Work and Health, Toronto, Canada; ${ }^{2}$ Monash University, Melbourne, Australia

\subsection{6/OEM-2019-EPI.43}

Time taken to first return to work (RTW) is often a primary endpoint for studies among injured workers. However, studies using administrative workers' compensation claims data have documented that a substantial proportion (approximately one half) of claimants will incur a subsequent period of wage replacement. Unfortunately, workers' compensation data is limited in the information collected to better understand which claimants are more likely to have a subsequent absence from work. The objective of this study is to address this gap using a cohort of workers' compensation claimants in the Australian state of Victoria.

The sample for this study is drawn from a longitudinal cohort of workers' compensation claimants $(n=869)$. For the purpose of this analysis we focused on those claimants who had returned to work (self-reported) at the baseline interview, which was conducted approximately 4 months after the injury had occurred $(n=372)$. Independent variables examined included if the respondent was working on full or partial duties, currently receiving health care for their injury, type of injury (musculoskeletal versus psychological), co-worker responses when they returned to work (measured using nine questions), and work limitations, measured using an abbreviated form of the work limitations questionnaire.

A total of 205 respondents (55\% of the sample) reported a subsequent absence from work when interviewed 6 months later. All independent variables, with the exception of injury type, were associated with subsequent absences from work. In a multivariable model, only working modified duties and greater limitations remained statistically significant.

The results of the current study help inform our understanding of trajectories in RTW and factors, measured after the first RTW, which may be associated with a subsequent absence from work. These findings can be integrated into RTW programs to help more workers achieve sustainable RTW following a work injury. 